\title{
SIMPATECTOMIA VIDEOTORACOSCÓPICA UNIPORTAL: UM AVANÇO DA TÉCNICA MINIMAMENTE INVASIVA NO Tratamento dA Hiperidrose Primária
}

\section{UNIPORTAL VIDEOTHORACOSCOPY SYMPATHECTOMY: AN ADVANTAGE IN THE MINIMALLY INVASIVE TECHNIQUE IN The Treatment of Primary Hyperhidrosis}

Julio Cesar Pereira Salustiano da Silva ${ }^{1}$, Maria Ribeiro Santos Morard ${ }^{1}$, Alfredo Jorge Vasconcellos Duarte $^{1}$, Alexandre Virla ${ }^{1}$, Pablo Magalháes ${ }^{1}$, Aline de Quadros Teixeira ${ }^{1}$, Thiago Scharth Montenegro ${ }^{1}$, Rossano Kepler Alvim Fiorelli1 ${ }^{2}$, Pietro Novellino ${ }^{1,2}$

${ }^{1}$ Serviço de Cirurgia Torácica - HUGG / Programa de Pós graduação em Medicina - Universidade Federal do Estado do Rio de Janeiro - UNIRIO

${ }^{2}$ Academia Nacional de Medicina

Autor correspondente:

Rossano Kepler Alvim Fiorelli

Email: fiorellirossano@hotmail.com

Tel: (21) 99973-2194

\section{RESUMO}

Introdução: A hiperidrose primária é caracterizada por uma sudorese excessiva sem etiologia definida, localizada primordialmente nas regióes palmar, plantar e axilar, de formas isoladas ou em associação. A simpatectomia videotoracoscópica é considerada como o melhor método terapêutico. A técnica uniportal pode resultar em melhor controle da dor, recuperação mais rápida e melhor resultado estético. Objetivo: Avaliar os resultados da Simpatectomia Videotoracoscópica 
Uniportal (SPTVU) e comparar com a técnica Multiportal (SPTVM) realizadas no Serviço de Cirurgia Torácica do Hospital Universitário Gaffrée e Guinle (SCTHUGG) da Universidade Federal do Estado do Rio de Janeiro (UNIRIO). Método: Estudo observacional, retrospectivo, descritivo, que avaliou pacientes portadores de hiperidrose primária que foram tratados no SCT-HUGG no período de agosto de 2004 a setembro de 2015. Foram analiadas diversas variáveis como gênero, idade, tipo da hiperidrose primária, nível de secção do nervo, tempo cirúrgico, complicaçóes pós-operatórias, controle da dor no pósoperatório e o grau de satisfação do paciente após a cirurgia. Resultados: Foram realizadas 52 operaçóes, sendo $42(80,8 \%)$ SPTVM e $10(19,2 \%)$ SPTVU. Quanto ao nível do bloqueio simpático nas duas técnicas houve o predomínio do bloqueio simpático no nível T3-T4. O tempo cirúrgico médio da SPVTU foi menor em relaçáo à média do tempo das SPVTM. A sudorese compensatória foi transitória e semelhante nos dois grupos. Não houve complicaçóes graves e nem óbito em nenhum dos grupos; não houve necessidade de pórtico adicional em nenhum dos casos do grupo SPTVU. Todos os pacientes alegaram um alto grau de satisfação e repetiriam a cirurgia. Conclusão: A simpatectomia vídeotoracoscópica uniportal foi uma modalidade terapêutica viável, eficaz e segura na amostra estudada.

\section{Palavra-chave}

Hiperidrose; simpatectomia; videotoracoscopia; uniportal; cirurgia torácica minimamente invasiva.

\section{ABSTRACT}

Introduction: Primary hyperhidrosis is characterized by excessive sweating beyond physiological needs without defined etiology, located primarily in the palmar and axillary regions, and may also affect the craniofacial region, in isolated or associated forms. Videothoracoscopic sympathectomy is considered as the best therapeutic method. The uniportal technique (single port) can result in better pain control, faster recovery and better aesthetic result. Objctive: To evaluate the results of the Uniportal videothoracoscopic sympathectomy (SPTVU) and compare it with the Multiportal technique (SPTVM) performed at the Department of Thoracic Surgery of the University Hospital Gaffrée e Guinle (SCT-HUGG) of the Federal University of Rio de Janeiro (UNIRIO). Method: This is a retrospective, descriptive, observational study in which the target population consisted of patients with 


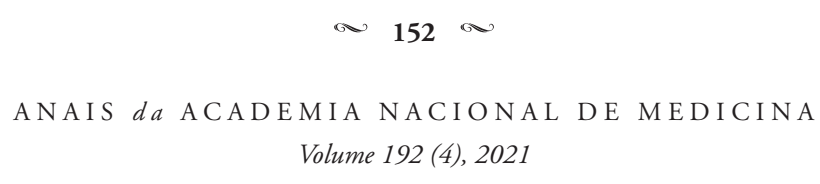

Volume $192(4), 2021$

primary hyperhidrosis who were treated in the SCT-HUGG from August 2004 to September 2015. The variables analysed were: gender, age, type of primary hyperhidrosis, nerve section level, surgical time, postoperative complications, control of paim in the postoperative period and the degree of patient satisfaction after surgery. Results: 52 surgeries were performed, $42(80,8 \%)$ SPTVM and $10 \quad(19,2 \%)$ SPTVU. As for the level of sympathetic block in the both techniques, there was also a predominance of sympathetic at T3T4 level. The mean surgical time of the SPTVU was shorther than the mean time of the SPTVM. Compensatory sweating was transient and similar in both groups. There were no serious complications or death in either groups; There was no need for additional portal in any of the cases of the SPTVU group. All the patients claimed a high degree of satisfaction and would repeat surgery. Conclusion: The uniportal videothoracoscopic Sympathectomy was a viable, effective and safe therapeutic modality in the sample studied.

\section{Keywords}

Hyperidrosis; sympathectomy; videothoracoscopy; single port; minimally invasive thoracic surgery.

\section{INTRODUÇÂOO}

A simpatectomia videotoracoscópica no tratamento da hiperidrose primária é um assunto em evidência atualmente, pois se trata de uma cirurgia relativamente recente para uma condição relativamente frequente. (1)

A hiperidrose é uma condição caracterizada pelo suor excessivo em desproporçáo à quantidade necessária para termorregulação e dissipação do calor corporal. (2) Pode ser generalizada, que envolve todo corpo e usualmente é parte de uma condição de base infecciosa, endócrina ou desordem neurológica ou primária também conhecida como essencial que é uma alteração de etiologia não completamente conhecida que envolve a estimulaçáo simpática excessiva pelo centro sudomotor na maioria dos casos. (3) Essa sudação excessiva é agravada por fatores emocionais e pelo calor. Com incidência superior a $1 \%$ na população, pode ocorrer apenas em uma região ou nas diversas associaçóes possíveis em locais como: palmas das mãos, axilas, pés, cabeça e face. (2-4)

É uma condição clínica que produz sérios constrangimentos ao seu portador, 
dificultando a execução das atividades diárias e interferindo no desempenho profissional, no lazer e nas atividades sociais. Esses indivíduos apresentam o centro hipotalâmico controlador do suor mais sensível aos estímulos emocionais oriundos das áreas corticais do cérebro. Os sintomas geralmente iniciam-se na infância ou na adolescência. $\mathrm{Na}$ maioria dos casos, essa sudação excessiva é precipitada por fatores emocionais e pelo calor. $(2,5,6)$ Existem vários tratamentos como: adstringentes tópicos, talcos absortivos, drogas anticolinérgicas, iontoforese, toxina botulínica e bloqueio percutâneo com uso de fenol. Entretanto, nenhum destes métodos mostrou-se capaz de reverter satisfatoriamente o problema, constituindo-se em métodos terapêuticos temporários. (1,7) Entre os tratamentos definitivos são citadas à lipossucção e a simpatectomia.(8) Com os avanços das técnicas videoendoscópicas, a simpatectomia por videotoracoscopia vem sendo realizada com uma frequência cada vez maior para o tratamento da hiperidrose, sendo este um procedimento minimamente invasivo. (9) Tal procedimento é considerado seguro e efetivo, aliando alto sucesso terapêutico com baixo risco de complicaçóes. Atualmente é considerado o padrão ouro no tratamento da hiperidrose. $(10,11)$

A simpatectomia videotoracoscópica consiste na secção, ressecção, ou clipagem da cadeia simpática torácica em níveis que podem variar de acordo com o objetivo almejado, não havendo consenso atual entre os níveis que seriam ideais para cada tipo de hiperidrose. De uma forma geral, pode-se correlacionar a secção em nível de T2 para hiperidrose craniofacial, T3 para palmar e T4 para axilar. A cirurgia é realizada, sob anestesia geral, através da introdução de um trocarte no tórax, pelo qual são inseridos a ótica acoplada à câmera de vídeo e os instrumentos, sendo o princípio desta cirurgia caracterizado pelo bloqueio dos gânglios simpáticos. (12)

A simpatectomia videotoracoscópica tem vantagens sobre as técnicas convencionais abertas, pois além de ser menos invasiva, fornece excelente exposição anatômica e visão magnificada das estruturas - fatores que facilitam na identificação da cadeia simpática. Embora atualmente o procedimento padrão realizado pela maioria dos serviços seja através da abordagem usando dois acessos (biportal), há um consenso crescente sobre a necessidade de aplicação de técnicas endoscópicas cada vez menos invasivas. Nesta perspectiva, o avanço da tecnologia vem permitindo a abordagem cirúrgica com apenas um acesso (uniportal). (13-15) Em geral, não são observadas complicaçóes perioperatórias ou necessidade de re-abordagem no pósoperatório imediato. As taxas de recidiva da hiperidrose após simpatectomia costumam ser baixas, em torno de 4\%. (16) 


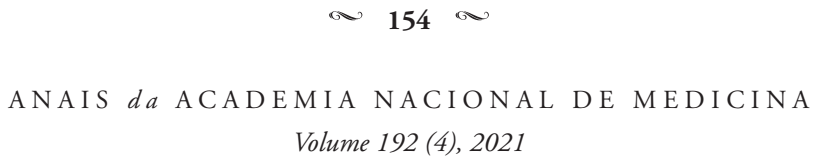

Volume 192 (4), 2021

A Equipe do Serviço de Cirurgia Torácica do Hospital Gaffrée e Guinle (HUGG) é uma referência no tratamento desta enfermidade, com uma larga experiência no uso da técnica desde 2004 e vem desenvolvendo a técnica uniportal (single port) há cerca de quatro anos, com excelentes resultados. (12)

\section{CASUÍSTICA E MÉTODO}

Trata-se de um estudo descritivo, observacional com análise retrospectiva em que foram incluídos os pacientes portadores de hiperidrose primária tratados no Serviço de Cirurgia Torácica do HUGG-UNIRIO no período de agosto de 2004 a setembro de 2015 . Os pacientes foram divididos em dois grupos: grupo I, aqueles submetidos à simpatectomia pela técnica uniportal (SPTVU) que vem sendo analisada prospectivamente desde 2012 e o grupo II (grupo histórico) aqueles submetidos à técnica multiportal (SPTVM) tradicionalmente empregada desde 2004.

Os dados foram coletados inicialmente a partir dos registros nos livros do SCTHUGG, complementados com os livros de registros do Centro Cirúrgico e analise sistemática dos prontuários requeridos ao
Serviço de Arquivos Médicos e de Estatística (SAME) do HUGG.

Os prontuários foram analisados em busca das variáveis estudadas e os dados coletados foram: gênero, idade, tipo da hiperidrose primária, nível da secção do nervo simpático, tempo cirúrgico, complicaçôes pós-operatórias, controle da dor no pósoperatório, grau de satisfação dos pacientes após a cirurgia e os resultados estéticos.

As informaçóes gerais sobre os pacientes estudados, incluindo a qualidade de vida antes e após o procedimento cirúrgico foram colhidas a partir de um questionário padronizado pelo Serviço. O questionário foi respondido pelos pacientes nos controles ambulatoriais e/ou por telefone e ficou anexado aos prontuários para as anotaçóes de folow up.

\section{Técnica cirúrgica da SPTVU}

O paciente sob anestesia geral, intubado com tubo de dupla luz tipo Robertshaw (intubação seletiva) para garantir a exclusão da ventilação pulmonar do lado que está sendo operado, é mantido em decúbito dorsal em posição semi sentada (semi Fowler) com o tronco elevado em 45 graus com dois coxins colocados longitudinalmente sob os ombros e o dorso; os braços ficam elevados e abduzidos a 90 graus e apoiados em braçadeiras da mesa 
operatória, procurando evitar distensão do plexo braquial; outro coxim é colocado sob os joelhos, na fossa poplítea permitindo uma posição fixa e confortavél. $\mathrm{O}$ procedimento cirúrgico é iniciado pelo lado direito, com uma incisão única de $1,5 \mathrm{~cm}$ na regiáo submamária, na altura do $5^{\circ}$ ou $6^{\circ}$, espaço intercostal na linha hemiclavicular (no sulco mamário nas mulheres e a $2 \mathrm{~cm}$ abaixo da borda areolar nos homens). Após a divulsão do plano muscular com uma pinça Kelly faz-se a toracostomia com trocarte de 11,5 $\mathrm{mm}$, por onde introduz a ótica de $5 \mathrm{~mm}$ de $0^{\circ}$ para visualização do tronco simpático. Pelo mesmo pórtico é introduzida a pinça Hook para realizar a secçáo do nervo nos níveis desejados: T2, T3 ou T4. A secção (simpaticotomia) éfeita na pleuramediastinal até o periósteo, sobre o arco costal, utilizando-se o eletrocautério monopolar ou eventualmente bisturi harmônico. Identificamos a segunda costela como a primeira visível no sentido craniocaudal e evitamos a seccáo neste nível para evitar o gânglio estrelado. Realizamos a secção no nível T2 apenas para os casos de hiperidrose craniofacial e rubor facial. Para os demais, realizamos essa secção sistemáticamente nos niveis T3-T4 e excepcionalmente englobamos o nível T5. Estendemos lateralmente a secção do periósteo costal em 03 a $04 \mathrm{~cm}$ visando seccionar ramos colaterais ou o nervo de Kuntz, caso estejam presentes. Um dreno torácico fino (16F) em selo d'água é colocado e retirado após a completa reexpansão pulmonar no final do procedimento. A seguir o procedimento é igualmente repetido no lado esquerdo. $\mathrm{O}$ controle radiológico é realizado no pós-operatório imediato e/ou antes da alta hospitalar.

\section{Técnica cirúrgica da SPTVM}

A técnica multiportal difere no número de pórticos adicionais que são colocados na linha axilar média na altura do $3^{\circ}$ ou $4^{\circ}$ espaço intercostal (biportal) e na axilar anterior (triportal). Pelos pórticos adicionais são intoduzidos os instrumentos para afastamento do pulmão, se necessário, secção de aderências, se houver, e a pinça Hook para secção do simpático.

\section{RESULTADOS}

De agosto de 2004 a fevereiro de 2015 foram realizadas 52 operações de SPTV, sendo $42(80,8 \%)$ das operaçóes pela técnica SPTVM e 10 (19,2\%) operações pela técnica SPTVU.

A casuística global foi constituída por 29 mulheres $(55,77 \%)$ e 23 homens 


\section{$\sim 156 \curvearrowright$

(44,23\%). No grupo da SPTVU foram 05 homens $(50 \%)$ e 05 mulheres $(50 \%)$ e no grupo SPTVM, 24 mulheres (46,15\%) e 18 homens (42,85\%).

A média geral de idade da população estudada foi de 28,20 anos sendo 29,04 anos de mulheres e 27,25 anos de homens. No grupo SPTVU a média de idade foi de 30,80 anos, sendo 33,20 para mulheres e 28,40 para homens. No grupo SPTVM a média foi de 27,80 anos, sendo 27,0 anos para mulheres e 29,05 anos para homens.

Quanto ao tipo da hiperidrose primária na população geral de 52 pacientes, houve um predomínio do tipo palmoplantar com 15 casos (28,8\%); seguiram-se os tipos palmar e axilar isolados com 10 casos $(19,2 \%)$ cada; palmo-axilar em 08 casos (15,03\%); palmo-plantar-axilar e palmoplantar-axilar com rubor facial em 04 casos $(7,6 \%)$ cada; craniofacial com rubor facial em 01 caso apenas $(1,9 \%)$.

No grupo SPTVU foram três casos (30\%) hiperidrose palmo-plantar, dois casos $(20 \%)$ do tipo axilar, três casos $(30 \%)$ palmar-axilar, um caso (10\%) palmar e um caso $(10 \%)$ craniofacial com rubor facial. No grupo SPTVM foi semelhante a predominância do tipo palmo-plantar com 12 casos (28,5\%), seguida pelo tipo palmar e axilar isoladas nove e oito casos $(21,4 \%$ e $16,6 \%)$, palmo-axilar cinco casos $(14,28 \%)$, palmo-plantar-axilar quatro casos $(9,52 \%)$, palmo-plantar-axilar-craniofacial três casos (7,14\%), palmo-plantar-axilar com rubor facial um caso $(2,38 \%)$.

Quanto ao nível do bloqueio simpático no grupo SPTVU predominou a secção no nível T3-T4 08 casos em (80\%); os níveis T2-T3 e T4-T5 em um caso (10\%) em cada. No grupo SPTVM também houve predomínio do bloqueio simpático no nível T3/T4 com 34 casos (80,95\%); a secção nos níveis de T2-T3-T4 foi realizada emcinco casos (11,90\%); os níveis de T2-T3 em dois casos (4,76\%) e T3-T4-T5 01 caso (2,38\%).

A média do tempo cirúrgico na SPTVU foi de 62,5 minutos, menor em relaçáo à média na SPTVM, que foi de 90,47 minutos. $\mathrm{Na}$ SPTVM, a técnica biportal foi de 78,42 minutos enquanto a técnica triportal foi de 103 minutos.

As limitaçóes sociais, afetivas e profissionais causadas pela hiperhidrose, foram relatadas em 100\% dos nossos pacientes. Dessas, 29\% foram classificadas como limitaçôes leves, $29 \%$ como moderadas e $42 \%$ como limitaçôes graves.

Não houve complicaçôes graves e nem óbito em nenhum dos grupos. Dos 10 casos do grupo SPTVU, nenhum necessitou pórtico adicional (conversáo para a técnica multiportal) e nem analgesia além da habitual, para o controle da dor no pósoperatório.

Do total de 52 pacientes, a nevralgia intercostal transitória foi relatada em quatro 
casos $(7,69 \%)$. Tivemos apenas um caso $(1,92 \%)$ de pneumotórax com permanência do dreno por mais de $48 \mathrm{~h}$. A sudorese compensatória foi relatada pela maioria dos pacientes $(81,8 \%)$ sendo este percentual semelhante em ambos os grupos e igualmente transitória. Ela foi relatada nas seguintes localizaçôes: face interna da coxa em 45,5 $\%$ dos casos, abdômen $36,4 \%$ casos, axilas em 36,4\% casos, tórax em 27,3\% dos casos, pés em 18,2\% dos casos e face em 9,0\% dos casos. A sudorese residual foi constatada em $19,23 \%$ entre os casos operados.

\section{DISCUSSÃO}

Nosso estudo abrangeu 52 pacientes portadores de hiperidrose primária que foram tratados pela simpatectomia torácica videotoracoscópica (SPTV) bilateral no período de agosto de 2004 a setembro de 2015. Destes pacientes, 42 (80,76\%) foram tratados pela técnica multiportal e $10(19,24 \%)$ pela técnica uniportal (SPTVU).

Com intuito de promover uma técnica ainda menos invasiva seguindo a tendência global, em 2012 passamos a realizar um estudo prospectivo náo randomizado visando desenvolver a técnica através de um único pórtico, com a prespectiva de melhorar os resultados pós-operatórios, tanto a nível de dor, tempo cirúrgico e resultado estético. Essa técnica uniportal portanto, foi o foco principal deste trabalho.

Ao avaliarmos os dados demográficos do nosso estudo, observamos um predomínio dos pacientes no sexo feminino $(55,77 \%)$ contra $44,23 \%$ no sexo masculino. Um estudo recente mostrou que $71,60 \%$ dos pacientes portadores de hiperidrose primária eram do sexo feminino enquanto $28,40 \%$ eram do sexo masculino. (10) Embora exista uma diferença percentual consideravél, os resultados do grupo geral de pacientes e do subgrupo de pacientes submetidos a SPTVM também mostraram uma prevalência pelo sexo feminino já no grupo da SPTVU náo houve diferença percentual entre os sexos, com 50\% de mulheres e $50 \%$ de homens. Ademais, a literatura atesta que a hiperidrose primária incide igualmente em ambos os sexos, porém, pelo o fato das mulheres serem mais suscetíveis aos estímulos psíquicos adversos e, consequentemente, procurarem tratamento com maior frequência, fica a impressão de que a hiperidrose predomina no sexo feminino.(17)

A idade média geral encontrada na nossa casuística foi em torno de 28,2 anos (variando de 13-59 anos). Observamos na literatura uma idade média geral de 32,4 anos, próxima da média de outros estudos expressivos. $(9,18)$ 
O tipo de hiperidrose primária representa o local do corpo em que se observam as manifestaçóes da sudorese excessiva. De uma maneira geral, nas axilas são afetadas em cerca de $51 \%$ dos pacientes, pés em 29\%, as palmas da mãos em $25 \%$ e a face em 20\%. (19) Outros estudos corroboram com a predominância do achado de sudorese excessiva nas axilas, em $66,7 \%$ dos casos, seguida pela palmar $21,6 \%$ e palmar-axilar 11,8\%. (9,20) Além desses estudos apontarem para o predomínio do acometimento de axilas e mãos, mostramnos também a falta de padronização para análise do tipo inicial da hiperidrose primária. Estudos recentes não levaram em conta tantos subtipos de associação da hiperidrose como fizemos em nosso trabalho, determinando assim menos subtipos desta disfunção. (17)

No presente estudo, além do local de maior intensidade de sudorese, classificamos também locais secundários em que os pacientes também se queixavam. Deste modo, nossos pacientes foram dividos náo somente quanto a um tipo de hiperidrose primária específica, mas em combinaçôes nos diversos tipos, quando presentes. No nosso estudo predominou o subtipo de hiperidrose palmo-plantar com 15 casos $(28,8 \%)$ e foi semelhante quando analisamos o grupo SPTVU com 30\% e SPTVM com 28,57\%; em seguida aparece a hiperidrose axilar e palmar com 19,23\%, estando em proporçóes semelhantes nos dois grupos. Corroborando a literatura, acreditamos que o acometimento principalmente das mãos e das axilas, onde a aparência é nitida pelo halo de suor nas roupas, leve os pacientes a maior constrangimento psicossocial e até mesmo laboral, levando a uma maior procura pelo tratamento. $(1,4,5,10,17,20)$ A evolução tecnológica dos materiais empregados possibilitou que houvesse um aperfeiçoamento da técnica cirúrgica, aumentando a segurança do procedimento e consequentemente, diminuindo a morbidade. Em vários serviços, a técnica evoluiu de uma abordagem unilateral, com intervalo de tempo de algumas semanas até se operar o lado oposto, para uma abordagem bilateral no mesmo ato cirúrgico, além da diminuição do número de trocartes utilizados. 21 A técnica cirúrgica prioritariamente utilizada hoje no Serviço de Cirurgia Torácica do HUGG consiste na abordagem uniportal bilateral e vem sendo analisada prospectivamente desde 2012.(12)

Os níveis dos bloqueios da cadeia simpática foram T3-T4, em mais de 80\% no grupo geral dos pacientes operados em ambas as técnicas. Nos casos de hiperidrose craniofacial ou rubor facial foi incluída a secção no nível de T2 como é estabelecido na literatura, por ser mais eficaz para esses tipos, embora sabidamente com maior incidência 
de sudorese compensatória. $(17,22)$ Como os tipos predominantes de sudorese em nossos pacientes incluíam as regiôes palmar e axilar, os níveis de bloqueios mais indicados foram em T3-T4. Em um paciente apenas realizamos o bloqueio nos níveis T4-T5 visando um melhor resultado. Entretanto náo podemos avaliar pela pequena casuística.

Quanto ao tempo cirúrgico, a média foi de 82,75 minutos quando somadas todas as técnicas, porém, constatamos que a técnica SPTVU conquistou o menor tempo cirúrgico, em torno de 62,5 minutos, quando comparada às outras técnicas SPTVM (bi ou triportal), com média de tempo 90,47 minutos. Observamos claramente que a otimizaçáo do tempo cirúrgico acompanha a evolução técnica de nossos procedimentos. A técnica uniportal, além de menos invasiva e de melhores resultados estéticos (uma única cicatriz submamária), consequentemente promove menor tempo de exposição do paciente à anestesia geral. $\mathrm{O}$ calibre da óptica que utilizamos $(5 \mathrm{~mm})$ pode eventualmente dificultar a manipulção dos instrumentos pelo mesmo pórtico. Apesar disso, nenhum dos casos necessitou pórtico adicional ou conversão para técnica multiportal. Para vencer essa dificuldade poderia ser utilizada uma óptica de $2 \mathrm{~mm}$, possivelmente proporcionando otimização ainda maior do procedimento, dado esse a ser comprovado em estudo futuro.
Em nossa casuística não tivemos nenhum óbito ou complicações expressivas. Tivemos apenas um paciente que permaneceu com dreno de tórax mais de $48 \mathrm{~h}$, necessitando de analgesia com opioide e quatro pacientes que tiveram nevralgia transitória no pósoperatório. Não foi aplicada escala de dor neste estudo. Consideramos a resposta positiva ou negativa ao uso da analgesia regular com dipirona e/ou anti-inflamatório náo esteroidal. Observamos que no grupo SPTVU nenhum paciente apresentou dor intensa. Todos tiveram controle da dor no local da incisão com analgesia comum. Complicações pósoperatórias são raras e acontecem em cerca de $1 \%$ das simpatectomias descritas. Dentre elas podem ocorrer: pneumotórax, hemotórax, sangramento de parede, infecção em sítio cirúrgico, nevralgia intercostal, dentre outras. (4)

Com relação a limitação social, afetivas e profissionais, ao indagarmos nossos pacientes a respeito das limitaçóes nestas esferas da vida que a disfunção causava impacto, $42 \%$ destes responderam que a hiperidrose primária impunha limitaçóes, afetivas e principalmente profissionais de intensidade "grave" em alguns casos culminando com demissão do trabalho. Os demais 58\% relataram que o grau de intensidade destas limitaçóes era "leve a moderada”. A partir disso, é possível inferir que parte dos nossos pacientes sofriam consideráveis consequências afetivas, sociais e profissionais. Alguns estudos usam outros 


\section{$\sim 160 \sim$

meios para avaliar a qualidade de vida dos seus pacientes, como escores. Em um estudo brasileiro, foi constatado que a média de qualidade de vida daquela população antes da simpatectomia era de 35,6 (em um escore de 0 a 100). 9 Apesar de utilizarmos meios diferentes para se avaliar o impacto da sudorese excessiva na qualidade de vida dos doentes, podemos notar que há um enorme impacto negativo na forma de o indivíduo com hiperidrose primária se relacionar em sociedade, o que pode implicar em consequências graves na vida dessas pessoas.

Dentro dessa amostra, 54,5\% afirmaram ter história familiar positiva para hiperidrose primária. A literatura nos mostra a presença de história familiar positiva que pode variar entre $30 \%$ a $54 \%$ dos pacientes portadores de hiperidrose primária. 10,23 Em nosso lenvatamento encontramos uma proporção dentro da média encontrada na literatura.

A sudorese residual teve uma prevalência baixa, $19,23 \%$ dos casos, enquanto a sudorese compensatória, aquela que surge em local antes não acometido, esteve presente em $81,8 \%$ dos casos. A despeito disso, todos os pacientes relataram satisfação imediata ao procedimento, uma vez que tanto a sudorese residual quanto a compensatória tinham uma intensidade muito inferior à hiperidrose primária prévia, culminando em um ganho considerável de qualidade de vida no pósoperatório imediato. Sobre a melhora da qualidade de vida e sua sustentação ao longo do tempo, Wolosker et al. concluiu que a melhora da qualidade de vida é imediata após a simpatectomia e se sustenta até o quinto ano.
Este mesmo autor relatou que 30 dias após a operação 90,0\% dos seus pacientes referiam melhora, 6\% relataram sudorese residual e que estavam na mesma condição prévia, e que ainda $3,7 \%$ relataram ter piorado. Ou seja, cerca de $10 \%$ de seus pacientes permaneceram com sudorese residual. (24)

A sudorese compensatória tem uma incidência de $84,3 \%$, segundo Baroncello et al. 9 Metanálises mostram que a sudorese compensatória está muito presente, atingindo em alguns estudos mais de 90\% dos pós-operatório imediato. 2,9 Logo, a nossa taxa de sudorese compensatória $(81,8 \%)$ converge com esses estudos entre outros da literatura.

Acreditamos que a continuidade deste estudo com aumento da casuística, vencimento da curva de aprendizagem e familiarização com a técnica uniportal poderá trazer dados mais expressivos e aplicáveis a prática clínica e futura padronização da técnica como mais uma opção terapêutica minimamente invasiva no tratamento definitivo da hiperidrose primária, no Serviço de cirurgia torácica do HUGG-UNIRIO.

\section{CONCLUSÃO}

A simpatectomia vídeotoracoscópica uniportal foi uma modalidade terapêutica viável, eficaz e segura na casuística estudada. Os resultados clínicos gerais foram semelhantes em ambas as técnicas, entretanto com vantagens para a técnica uniportal no 
que se refere ao tempo cirúrgico, ao controle da dor pós-operatória e ao resultado estético.

\section{REFERÊNCIAS BIBLIOGRÁFICAS}

1. Doolabh N, Horswell S, Williams M, et al. Thoracoscopic sympathectomy for hyperhidrosis: indications and results. The Annals of thoracic surgery. 2004;77(2):410-414.

2. Haider A, Solish N. Focal hyperhidrosis: diagnosis and management. Cmaj. 2005;172(1):69-75.

3. Raia AA, Zerbini EdJ. Clínica cirúrgica Alípio Correa Netto. In: Clínica cirúrgica Alípio Corrêa Netto.1988:914-914.

4. Bejarano B, Manrique M. Simpatectomía toracoscópica: una revisión de la literatura. Neurocirugía. 2010;21(1):5-13.

5. Matthews BD, Bui HT, Harold KL, et al. Thoracoscopic sympathectomy for palmaris hyperhidrosis. Southern medical journal. 2003;96(3):254-259.

6. Ro KM, Cantor RM, Lange KL, Ahn SS. Palmar hyperhidrosis: evidence of genetic transmission. Journal of vascular surgery. 2002;35(2):382-386.

7. Ayres M, Júnior Aires M, Ayres DL, Santos AdAS. Bio Estat 5.0: aplicaçóes estatística nas áreas das ciências biológicas e médicas. In: Bio Estat 5.0: aplicaçôes estatística nas áreas das ciências biológicas e médicas.2007:364-364.

8. Dias L, Marçal L, Rodrigues M, Alves TC, Pondé MP. Eficácia da toxina botulínica no tratamento da hiperidrose. Revista Neurociências. 2001;9(3):9396.

9. Baroncello JB, Baroncello LRZ, Schneider EGF, Martins GG. Evaluation of quality of life before and after videothoracoscopic simpathectomy for primary hyperhidrosis. Revista do Colégio Brasileiro de Cirurgióes. 2014;41(5):325-330.

10. Atkinson JL, Fode-Thomas NC, Fealey RD, Eisenach JH, Goerss SJ. Endoscopic transthoracic limited sympathotomy for palmar-plantar hyperhidrosis: outcomes and complications during a 10-year period. Paper presented at: Mayo Clinic Proceedings2011.

11. Munia MAS. Comparação dos resultados obtidos no tratamento da hiperidrose axilar pela simpatectomia torácica videotoracoscópica nos níveis de desnervação: T3-T4 versus T4, Universidade de São Paulo; 2010.

12. Fiorelli RKA, Elliot LG, Alvarenga RMP, et al. Avaliação do impacto na qualidade de vida de pacientes portadores de hiperidrose primária submetidos à simpatectomia videotoracoscópica. Revista Meta: Avaliação. 2011;3(7):124.

13. Gossot D, Kabiri H, Caliandro R, Debrosse D, Girard P, Grunenwald D. Early complications of thoracic endoscopic sympathectomy: a prospective study of 940 procedures. The Annals of thoracic surgery. 2001;71(4):1116-1119. 
14. Lardinois D, Ris HB. Minimally invasive video-endoscopic sympathectomy by use of a transaxillary single port approach. European journal of cardio-thoracic surgery. 2002;21(1):67-70.

15. Vanaclocha V, Sáiz-Sapena N, Panta F. Uniportal endoscopic superior thoracic sympathectomy. Neurosurgery. 2000;46(4):924-928.

16. Martinez-Barenys C, Pérez J, De Castro $\mathrm{PL}$, et al. Initial experience of a program of clipping the sympathetic nervous system for the treatment of hyperhidrosis and facial flush. Cirugía Española (English Edition). 2013;91(2):115-120.

17. Kauffman P, de Campos JRM, Wolosker N, Kuzniec S, Jatene FB, Leão PP. Simpatectomia cervicotorácica videotoracoscópica: experiência de 8 anos. Jornal Vascular Brasileiro. 2020;2(2):98-104.

18. Baumgartner FJ. Surgical approaches and techniques in the management of severe hyperhidrosis. Thoracic surgery clinics. 2008;18(2):167-181.

19. González MAP, Serdá GJ, Suarez PR, Perez-Peñate G, Gilart JF, Navarro PC. Long-term cardiopulmonary function after thoracic sympathectomy: Comparison between the conventional and simplified techniques. The Journal of thoracic and cardiovascular surgery. 2010;139(2):405-410.

20. Nicoleit AR, Psendziuk C, Galvani GdC, Pereira SW, Kestering DdM. Videosimpatectomia torácica para tratamento da hiperidrose primária. Arquivos Catarinenses de Medicina. 2009;38(3):24-31.

21. Boscardim PCB, Oliveira RAd, Oliveira AAFRd, Souza JMd, Carvalho RGd. Simpatectomia torácica ao nível de $4^{\mathrm{a}}$ e $5^{\mathrm{a}}$ costelas para o tratamento de hiper-hidrose axilar. Jornal Brasileiro de Pneumologia. 2011;37(1):6-12.

22. Leão LEV, Giudici R. Simpatectomia Torácica por Videotoracoscopia: tratamento atual da hiperhidrose palmar. Revista Jovem Médico. 1999;3(2).

23. Strutton DR, Kowalski JW, Glaser DA, Stang PE. US prevalence of hyperhidrosis and impact on individuals with axillary hyperhidrosis: results from a national survey. Journal of the American Academy of Dermatology. 2004;51(2):241-248.

24. Stolman LP. Treatment of hyperhidrosis. Dermatologic clinics. 1998;16(4):863869. 